Caso clínico

\title{
Epilepsia focal asociada a tumor cerebral
}

\section{Focal epilepsy associated with brain tumor}

\section{Peña, Susana}

\author{
Susana Peña \\ supeamart@yahoo.com \\ Hospital Nacional Rosales, El Salvador
}

\author{
Alerta \\ Ministerio de Salud, El Salvador \\ ISSN-e: 2617-5274 \\ Periodicidad: Semestral \\ vol. 1 , núm. 2, 2018 \\ ralerta@salud.gob.sv \\ Recepción: 02 Marzo 2018 \\ Aprobación: 16 Marzo 2018 \\ Publicación: 12 Diciembre 2018 \\ URL: http://portal.amelica.org/ameli/journal/419/4191909013/ \\ DOI: https://doi.org/10.5377/alerta.v1i2.7137
}

Citación recomendada: Peña S. Epilepsia focal asociada a tumor

cerebral. Alerta. 2018;1(2):87-90. DOI: 10.5377/alerta.v1i2.7137

\section{RESUMEN}

La epilepsia es una enfermedad neurológica crónica frecuente. La Organización Mundial de la Salud (OMS) ha reportado, a la fecha, cerca de 50 millones de casos en el mundo, de los cuales el $85 \%$ proviene de países en desarrollo ${ }^{1}$. Según el origen de la actividad epiléptica se clasifica en focal y generalizada; según la etiología, en

estructural, genética, infecciosa, metabólica o inmune ${ }^{2}$. Se describe caso de mujer de 38 años, con epilepsia de larga evolución, aumento reciente de frecuencia de crisis epilépticas, con evidencia en neuroimagen por resonancia magnética de neoplasia cerebral, confirmando origen focal estructural de epilepsia.

\section{INTRODUCCIÓN}

La epilepsia, por definición, es la ocurrencia de dos o más crisis epilépticas no provocadas ${ }^{3}$. Puede ser focal o generalizada ${ }^{4}$. Las crisis epilépticas de tipo focal se originan de sitios específicos en un hemisferio, con o sin propagación contralateral ${ }^{4}$. Entre las causas de epilepsia de tipo focal se debe valorar la edad de aparecimiento de la primera crisis, para considerar entre ellas causas estructurales, como la formación de neoplasias. Cuando se identifica causa estructural como origen de la actividad epiléptica se denomina epilepsia focal estructural ${ }^{4}$.

Una de las causas de crisis epilépticas son las neoplasias 5 . Las manifestaciones clínicas se correlacionan al sitio donde se encuentra la lesión neoplásica, según descriptores clínicos motores, sensitivos y de 
automatismos, entre otros, con o sin generalización secundaria ${ }^{6}$. Es frecuente que este tipo de epilepsia sea refractaria, siendo necesario el uso de más de dos antiepilépticos a dosis altas ${ }^{7}$.

Se ha establecido, además, el término de "tumores asociados a epilepsia crónica", con el que se describen pacientes con historia de epilepsia de larga evolución, de aparecimiento en edades jóvenes, de carácter usualmente focal, de tipo refractario, con identificación de lesiones neoplásicos por neuroimagen. Al ser sometidos a resección quirúrgica, se encuentran hallazgos histopatológicos de tipos bien diferenciados. Son tumores de crecimiento lento, de larga supervivencia, con localización preferencial en regiones temporales, sobre todo en el hipocampo ${ }^{8}$.

El Hospital Nacional Rosales es el centro nacional especializado de referencia, donde la epilepsia está entre las primeras causas de consultas neurológicas, por lo que su identificación oportuna es vital para un adecuado manejo.

El promedio de consultas anuales osciló entre 3000 a 4000 pacientes durante el año 2016, según el Sistema de Morbimortalidad en Líinea (SIMMOW) del Ministerio de Salud ${ }^{9}$. El tipo de epilepsia idiopático generalizado es el más frecuentemente reportado, y en segundo lugar, el tipo focal sintomático?

\section{Presentación del caso}

Mujer de 38 años, con historia de inicio de crisis epilépticas a los 8 años de edad, descritas como movimientos tónicos-clónicos generalizados, de duración variable entre dos y cinco minutos, a predominio nocturno, precedidos en ocasiones de arresto de conciencia y automatismos manuales. Tratamiento recibido en hospital periférico: fenitoina $100 \mathrm{mg}$ vía oral cada 8 horas. Las crisis aumentan durante los últimos tres años, siendo hasta de 5-6 por mes, por lo que es referida al Hospital Nacional Rosales. Al examen físico: no se identifica déficit neurológico. Se realiza Video Electroencefalograma (EEG) y Resonancia Magnética (RMN) cerebral (Figura 1). En el estudio de neuroimagen se identificó anomalía estructural de tipo "lesión córtico subcortical temporal izquierda hipointensa en fases de imagen potenciada en $\mathrm{T} 1$ y en fase de recuperación de inversión atenuada de fluido (FLAIR), con aspecto hiperintenso en imagen potenciada en T2 con áreas quísticas en su interior, que no realza con gadolinio, ni restringe en difusión", descrita por radiología como sospecha de glioma de bajo grado vs. tumor neuroepitelial disembrioblástico. En el video EEG se correlacionó dicha lesión con actividad epiléptica con poli puntas de inicio temporal izquierdo, propagación contralateral y generalización secundaria. En evaluación por neurología se agrega al tratamiento farmacológico ácido valproico $500 \mathrm{mg}$ vo cada 8 horas, con reducción de frecuencia de las crisis a 1 a 2 por mes.

Figura 1. A y B) En RMN lesión córtico subcortical temporal izquierda hipertensa en T2 e hipointensa en FLAIR, con áreas quísticas en su interior, que no realza con gadolinio, ni restringe en difusión. C) En espectroscopía se evidencia elevación de colina y disminución de n-acetil aspartato (NAA). Posibilidad a considerar glioma de bajo grado vs. tumor neuroepitelial disembrioblástico. Fuente: Servicio de Radiología e Imágenes/Hospital Rosales.

\section{Discusión}

La paciente descrita ha presentado una larga evolución de epilepsia, con aumento de frecuencia de las crisis epilépticas. Al identificar lesión neoplásica en región temporal izquierda por resonancia magnética se documenta origen estructural de la epilepsia. Es candidata a estudio neuroquirúrgico e histopatológico para identificar estirpe específico de neoplasia de lóbulo temporal para planificar abordajes.

Este tipo de neoplasias ha sido reportado con mayor frecuencia a nivel frontal y temporal, con un lento crecimiento neococortical y los tipos histológicos reportados son gangliogliomas y tumores neuroepiteliales 
disembrioblásticos ${ }^{8}$. El tratamiento quirúrgico ha reportado control de crisis epilépticas en la mayoría de los $\operatorname{casos}^{10}$.

El abordaje clínico de un paciente con diagnóstico de epilepsia focal siempre debe iniciar con una historia clínica detallada que comprenda todos aquellos descriptores clínicos que permitan identificar probables zonas epileptógenas como origen focal ${ }^{5}$. Luego, el EEG convencional se debe complementar con un video EEG para correlacionar la actividad eléctrica con la actividad clínica epiléptica y así determinar el origen de dicha zona epiléptica. Para identificar causas epilépticas estructurales, el estudio de neuroimagen de elección es la resonancia magnética, con énfasis en lóbulos temporales ${ }^{11}$.

\section{ConCLusión}

La epilepsia es una enfermedad frecuente en todos los niveles de atención en El Salvador, que debe alertar al médico cuando es focalizada o se vuelve refractaria, obligando a realizar estudios complementarios que permitan identificar una causa estructural. Una vez identificada, se procede al manejo específico. Este caso refleja que ante el cambio de patrón de crisis epilépticas se debe considerar causa estructural de la refractariedad.

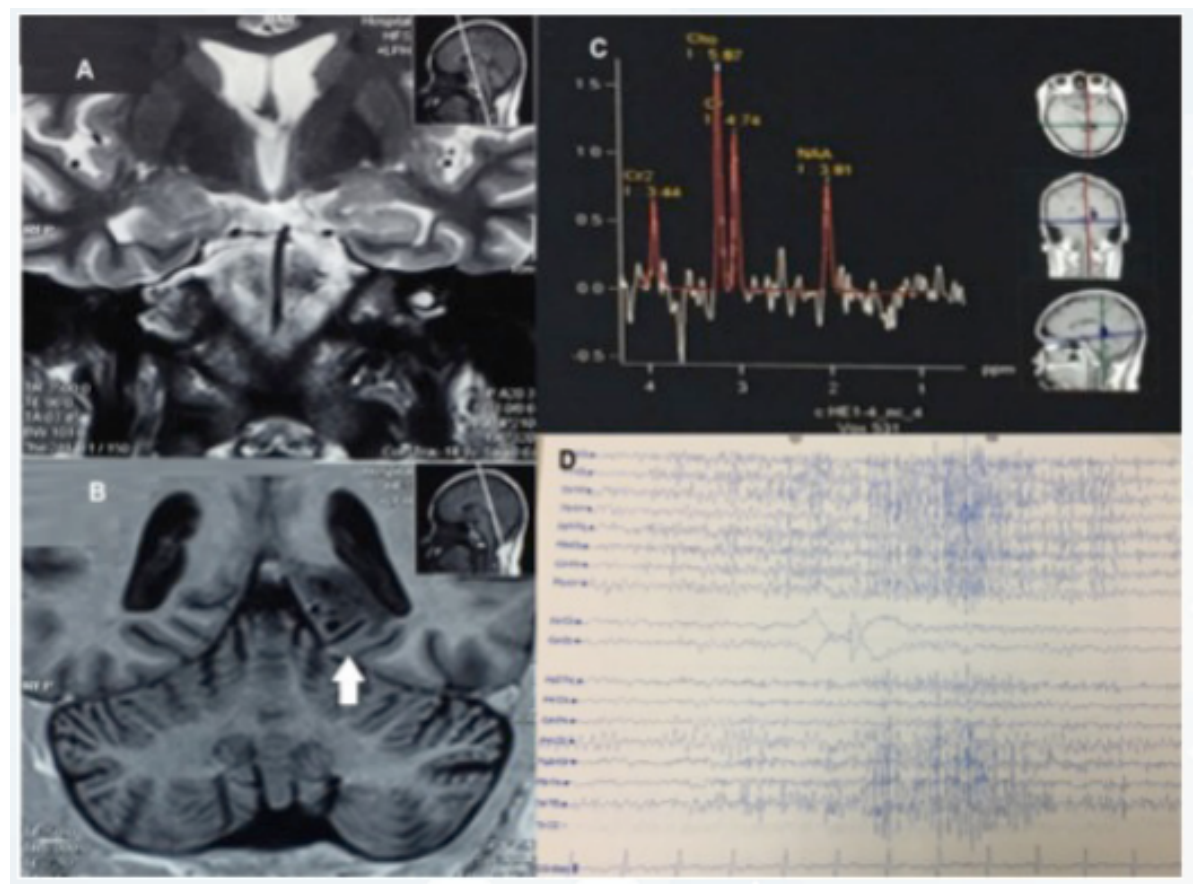

FIGURA 1

A y B) En RMN lesión córtico subcortical temporal izquierda hipertensa en T2 e hipointensa en FLAIR, con áreas quísticas en su interior, que no realza con gadolinio, ni restringe en difusión. C) En espectroscopía se evidencia elevación de colina y disminución de n-acetil aspartato (NAA). Posibilidad a considerar glioma de bajo grado vs. tumor neuroepitelial disembrioblástico. D) Video EEG: actividad epiléptica con poli puntas de inicio temporal izquierdo con propagación contralateral y generalización secundaria. Servicio de Radiología e Imágenes/Hospital Rosales (A,B,C) y Unidad de Neurofisiología/Hospital Rosales (D) 


\section{REFERENCIAS BIBLIOGRÁFICAS}

1. Organización Mundial de la Salud (OMS). Acceso/Centro de prensa/Notas descriptivas/Epilepsia. Consultado 26 de febrero 2018. Washington D.C. Disponible en: http://www.who.int/es/news-room/fact-sheets/detail/epil epsy

2. Berg A. Revised terminology and concepts for organization of seizures and epilepsies: Reports of the ILAE Commission on Classification and Terminology, 2005-2009. Epilepsia. 2010; 51(4):676-685.

3. Fisher R. A practical clinical definition of epilepsy. Epilepsia. 2014; 55(4):475-482.

4. Sheffer I. ILAE classification of the epilepsies: Position paper of the ILAE Commission for Classification and Terminology. Epilepsia. 2017; 58(4):512-521.

5. Holthausen H, Blumcke I. Epilepsy associated tumors: what epileptologists should know about neuropathology, terminology and classification systems. Epileptic Disord. 2016; 18(3): 240-51.

6. Fisher RS. Operational classification of seizure types by the International League against Epilepsy: Position paper of the ILAE Commission for Classification and Terminology. Epilepsia. 2017; 58(4): 522-530.

7. Blumcke I, Aronica E, Urbach H, Alexopoulos A, González M J. A neuropathology based approach to epilepsy surgery in brain tumors and proposal for a new terminology use for long term epilepsy associated to brain tumors. Acta Neuropathol. 2014; 28: 39-54.

8. Luyken C. The spectrum of long term epilepsy associated tumors: long term seizure and tumor outcome and neurosurgical aspects. Epilepsia. 2003; 44(6):822-830.

9. Ministerio de Salud de El Salvador. Sistema de Morbimortalidad on line (SIMMOW). Consultado el 26 de febrero 2018. San Salvador. Disponible en: http://simmow.salud.gob.sv

10. Englot DJ, Berger MS, Barbaro NM, Chang EF. Factors associated with seizure freedom in the surgical resection of glioneuronal tumors. Epilepsia. 2012; 53(1):51-7.

11. Duncan J, Tisis J. MRI in the diagnosis and management of epileptomas. Epilepsia. 2013; 54(Suppl.9): 40-43. 